\title{
Impeller Design Using CAD Techniques and Conformal Mapping Method
}

\author{
Milos Teodor \\ "Politehnica" University of Timisoara, \\ Romania
}

\section{Introduction}

Computerized pump design has become a standard practice in industry, and it is widely used for both new designs as well as for old pumps retrofit. Such a complex design code has been developed over the past decade by the author. However, any design method has to accept a set of hypotheses that neglect in the first design iteration the three-dimensional effects induced by the blade loading, as well as the viscous effects. As a result, an improved design can be achieved only by performing a full 3D flow analysis in the pump impeller, followed by a suitable correction of the blade geometry and/or the meridian geometry. This chapter presents a fully automated procedure for generating the inter-blade channel 3D geometry for centrifugal pump impeller, starting with the geometrical data provided by the quasi-3D code. This is why we developed an original procedure that successfully addresses all geometrical particularities of a centrifugal pump.

\section{Domain generation of axial-symmetric flow in impeller area}

Fluid movement in the impeller area is axially symmetric. The reference system adequate to this kind of flow is cylindrical $(r, \theta, z)$. Because of geometric axial symmetry of the domain area we have axial-flow symmetry and cinematic, so the study of spatial movement can be reduced to the study of plane movements, or in a meridian plane, plane containing the rotation axis of symmetry, $\mathrm{Oz}$. The resolution must be analytical in order to continue to analytically generate the mesh network to simulate the flow by Finite Element Method (FEM). The inlet data are the main dimensions of the preliminary study. First axial and radial extensions should be set. Axial extension, fig. 1, shall be determined based on previous studies (Gyulai, 1988) relation:

$$
z_{\max }=1,1 \cdot D_{0}
$$

Radial expansion is taken by $25 \%$ over $D_{2 e x}$ diameter $\left(D_{2}\right.$-extended). At the mixed-flowimpellers, impeller diameter output, $D_{2}$, is in the area of transition from axial to radial movement. Therefore the domain must be extended to at least $2 D_{0}$. So if $D_{2}<2 D_{0}$ then $D_{2 e x}=2 D_{0}$, and if $D_{2} \geq 2 D_{0}$, then $D_{2 e x}=D_{2}$. From $D_{2 e x}$ we calculate $D_{2 \max }$ with the relationship:

$$
D_{2 \max }=1,25 \cdot D_{2 e x}
$$




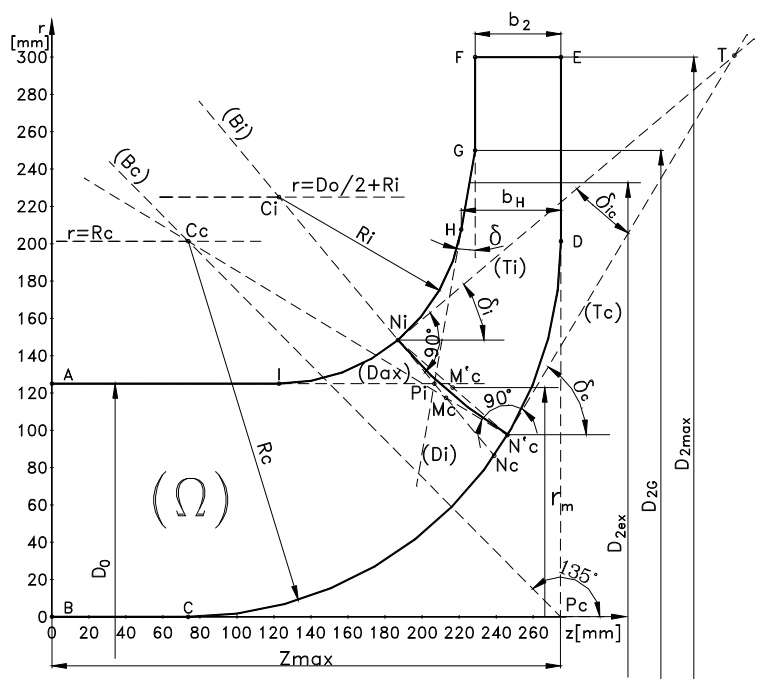

Fig. 1. Geometric construction of half-domain of flow

$Z_{\max }$ and $D_{2 \max }$ define dimensions of the gauge domain. Next axial area with radial area is connected. Because of technological reasons the shroud connection is made with a straight segment $G H$ and an arc of radius $R_{i}$ and at the hub only an arc of radius $R_{c}$. Input section $A B$ is determined by the input diameter $D_{0}$ and output section $E F$ of diameter $D_{2 \max }$ and width $b_{2}$. First the shroud connection is done by and depending of its position is determined the hub radius $R_{c}$ and its centre position. The starting point is $G$ at $D_{2 G}=1,05 D_{2 e x} . D_{2 G}$ is placed by $5 \%$ above $D_{2 e x}$ because if $D_{2 e x}=D_{2}$ then in the vicinity of $G$ appear some inflections in meridian speeds variation which will distort the speed triangles of output. From $G$ goes straight $(D i)$ tilted from the vertical at angle $\delta=2^{\circ} \ldots 8^{\circ}$. Angle $\delta$ is chosen in an initial approximation for $n_{q}$-low around $2^{\circ}$, and for $n_{q}$-high values close to $8^{\circ}$. Many solutions are possible within the range specified; it is chosen the one taking into account other considerations, then calculating the required width at $D_{2 e x}$ diameter. It is usually opted for a rounded value, and after obtaining velocity variation along the streamlines the calculation can be redone with other values of the tilt angle $\delta$ of conical area.

From $A$ doing a parallel to $O z$ right results straight (Dax), which intersected with $(D i)$ determines the point $P_{i}$. The centre of connecting arc HI will be on bisector of angle. $R_{i}$ is chosen depending on the type of impeller, for slow impellers, $n_{q}$-low, $R_{i}$ is small, and for fast impellers, $n_{q}$-high, is high because the impeller's blades will be mainly in the area of curvature (crossing).

Exact coordinates of point of contact $H$ result from the analytical condition of tangent for the straight taken from point $G$ to the arc connecting the shroud, for all using polar equation and tangent equation to a circle of analytical geometry.

Shroud area is completely defined, the following step will be to determine the hub radius of the arc connecting to the passage so that the section area of curvature equals to the input section. Centre of arc connecting the hub will be on bisector $(B C)$ of right angle $C \hat{P}_{c} D$. 
Finding the optimal connection radius is done through repeated testing, starting with low values until the condition of equal sections is verified with an error less than $1 \%$.

The passage sectional area is calculated and verified in the end by the relationship:

$$
A_{m}=2 \pi r_{m} b_{m}=\frac{\pi D_{0}^{2}}{4}
$$

This way the domain is completely defined analytically and with the exception of point G, all connections are continuous and crossing sections from A to $\mathrm{G}$ are relatively constant.

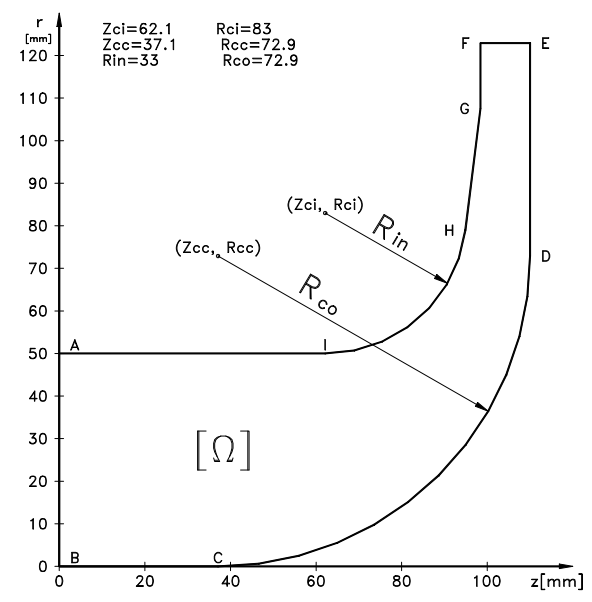

a) $\mathrm{n}_{\mathrm{q}}=25,7$

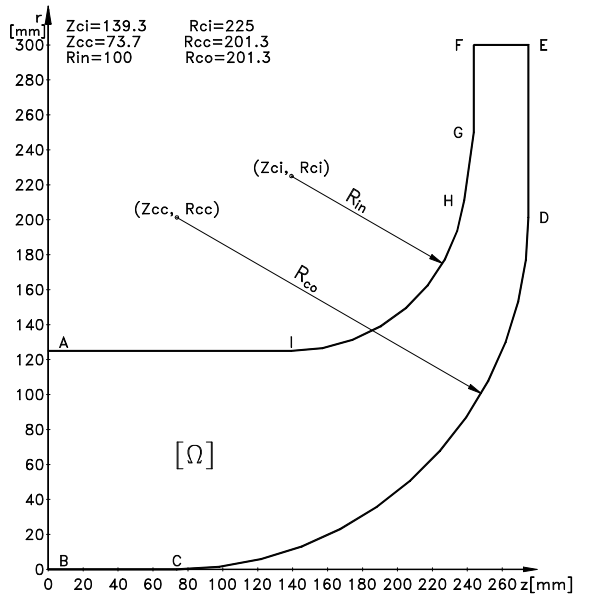

b) $\mathrm{n}_{\mathrm{q}}=80,8$

Fig. 2. Analyzing domain with finite elements

\section{Domain discretization}

Integrating flow function $\psi$ and potential velocity function $\varphi$ is done by FEM using quadrilateral izo-parametric linear finite elements. To maintain good accuracy in the application of FEM it is necessary that the sides of quadrilaterals to be relatively equal in length, that some sides are not disproportionately small compared to others.

Since the average length and width ratio of the domain is about 10, 100 intervals were taken between input and output and 10 intervals in the cross section. Borders shroud and hub were divided into 100 equal intervals resulting in 101 points (Fig. 3). Homologous points on the shroud and hub were joined together by line segments. Each straight segment in turn was divided into 10 intervals, resulting in 11 points on each segment. Number of nodes of the mesh was $101 * 11=1111$, every 4 neighbouring nodes create an irregular quadrilateral that will be the base of integration for stream function $\Psi$ in partial differential equation.

This method is recommended for the case when the mesh is an exercise in applying FEM. Flow professional software automatically solves this problem after the domain geometry is defined. 


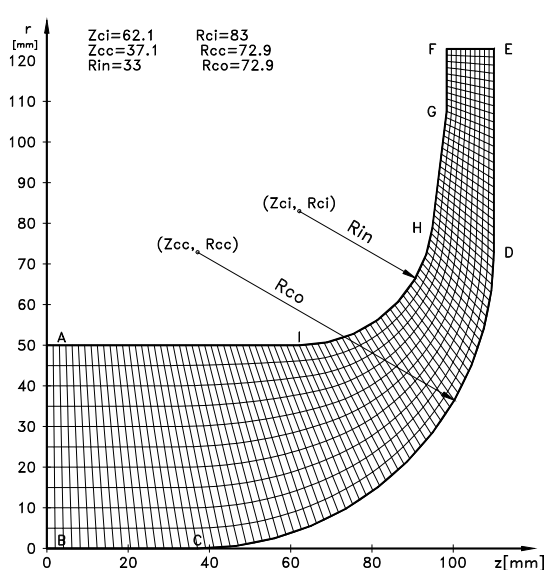

a) $n_{q}=25,7$

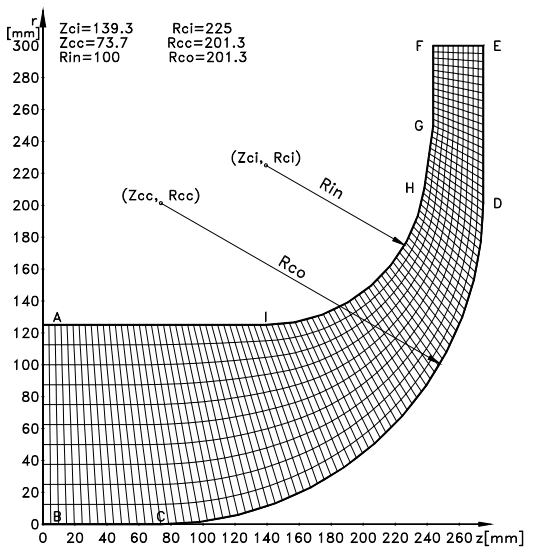

b) $\mathrm{n}_{\mathrm{q}}=80,8$

Fig. 3. Network of the finite element mesh

\section{Hydrodynamic field of the impeller}

Partial differential equation of the stream function $\psi$ is a Helmholtz equation of elliptic cylindrical coordinates:

$$
\frac{\partial^{2} \psi}{\partial z^{2}}+\frac{\partial^{2} \psi}{\partial r^{2}}-\frac{1}{r} \frac{\partial \psi}{\partial r}=0
$$

Flow differential function is:

$$
\mathrm{d} \psi=\frac{\partial \psi}{\partial r} \mathrm{~d} r+\frac{\partial \psi}{\partial z} \mathrm{~d} z=r v_{z} \mathrm{~d} r-r v_{r} \mathrm{~d} z
$$

where velocity components are:

$$
v_{z}=\frac{1}{r} \frac{\partial \psi}{\partial r} \quad ; \quad v_{r}=-\frac{1}{r} \frac{\partial \psi}{\partial z}
$$

Integrating this equation is done by FEM using the real values of coordinates of the points of the mesh network, expressed in mm. Dimensionless treatment has been dropped because of the method in which concrete cases are solved for the later stages using real values of velocities. Solving the global FEM system is better than unknowns, values of function $\psi$ to be of the same order of magnitude with coefficients given by the coordinates of points in order to increase accuracy of calculation. Therefore $\psi$ admits border values between 0 and 100 .

\section{The boundary conditions on domain border}

Borders AIHGF and BCDE are streamlines and $\psi=$ const along them. We recognize on AF boundary $\psi=100$ and on $\mathrm{BE}, \psi=0$. Thus on input boundary we have a uniform flow with constant velocity on the entire section: 


$$
v_{r}=0 ; v_{z}=\frac{4 Q}{\pi D_{0}^{2}}
$$

Replacing (7) in (5) and integrating results:

$$
\psi=\int \frac{4 Q}{\pi D_{0}^{2}} r \mathrm{~d} r=\frac{4 Q}{2 \pi D_{0}^{2}} r^{2}+C_{i}
$$

Admitting that on the hub border we have $\psi=0$, including the point $B$, will result integration constant $C_{i}$ :

$$
(r=0) \Rightarrow(\psi=0) \Rightarrow\left(C_{i}=0\right)
$$

On the shroud border stream function will be constant and equal to that of point $A$ where $r=D_{0} / 2$, and $\psi=Q / 2 \pi=100$ (quasi-unitary flow).

$$
v_{r}=\frac{Q}{\pi D_{2 \max } b_{2}} ; v_{z}=0
$$

Replacing (4.10) in (4.5) and integrating results:

$$
\psi=-\int r v_{r} \mathrm{~d} z=-\left.\int r \frac{Q}{\pi D_{2 \max } b_{2}} \mathrm{~d} z\right|^{r=\frac{D_{2 \max }}{2}}=-\frac{z Q}{2 \pi b_{2}}+C_{e}
$$

Admitting the same conditions on the solid boundaries result:

$$
\left(z=z_{E}\right) \Rightarrow(\psi=0) \Rightarrow\left(\mathrm{C}_{\mathrm{e}}=\frac{Q z_{\max }}{2 \pi b_{2}}\right)
$$

As a result, the law of variation $\psi$ on the border $E F$ is given by:

$$
\psi=-\frac{Q z}{2 \pi b_{2}}+\frac{Q z_{\max }}{2 \pi b_{2}}=\frac{Q}{2 \pi} \frac{1}{b_{2}}\left(z_{\max }-z\right)
$$

Integration with FEM of the Helmholtz equation (4) will be in an area where function values are imposed on border which means that we have to solve a problem of Dirichlet type.

\section{Calculus of stream function $\Psi$ by FEM}

The above have created all necessary conditions for the Helmholtz equation (4) integration by FEM. Function $\psi$ can be globally approximated on $\Omega$ by:

$$
\psi=a_{\alpha} \psi_{\alpha} ; \alpha=\overline{1, \mathrm{G}}
$$

where $G$ is the number of nodes on $\Omega$. Applying Galerkin's method (Anton at al. 1988) follows:

$$
\int_{\Omega}\left(\frac{\partial^{2} \psi}{\partial z^{2}}+\frac{\partial^{2} \psi}{\partial r^{2}}-\frac{1}{r} \frac{\partial \psi}{\partial r}\right) a_{\alpha} \mathrm{d} \Omega=0
$$


Using the notation (Anton at al. 1988) resolution is reduced to solving the global linear system of equations:

$$
D_{\alpha \beta} \psi_{\beta}=F_{\alpha} \text { where } \alpha, \beta=\overline{1, G}
$$

The method of solving the system can be Gauss-Seidel iterative method, resulting in final values of the stream function $\psi$ mesh nodes. From a mathematical point of view the lines are defined by the geometrical locus of points where the stream function has the same values. If between the solid borders the stream function $\psi$ takes values between 0 and 100, then streamlines having $\psi=10,20, \ldots, 90$ are looked for because $\psi=0$ and $\psi=100$ are the solid borders. Identification of the points for stream lines is made through interpolation with cubic SPLINE function to have more precision.

Applying the same methodology (Anton at al. 1988) as for the stream function $\psi$ can integrate the equation for the velocity potential function, $\varphi$, in the end getting equipotential lines that overlap the stream lines as shown in Fig. 4.

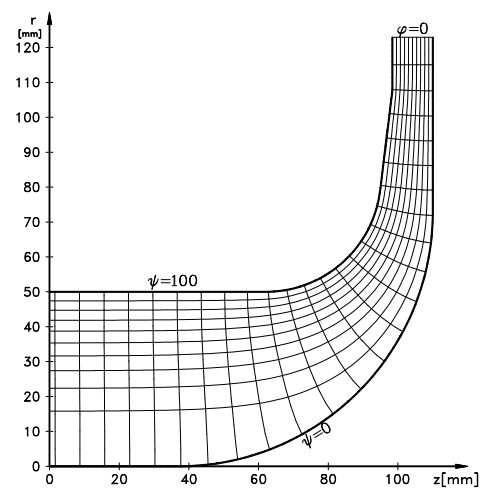

a) $\mathrm{n}_{\mathrm{q}}=25,7$

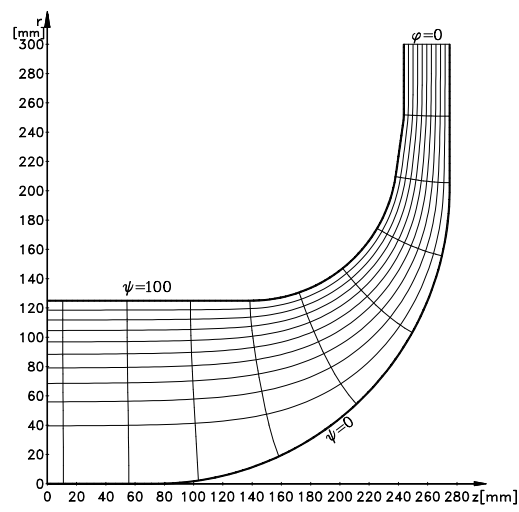

b) $\mathrm{n}_{\mathrm{q}}=80,8$

Fig. 4. Stream and equipotential lines of the hydrodynamic spectrum

\section{Determination of the velocities and pressures fields}

Taking into account relations (6) and the notation (Anton at al. 1988) meridian velocity components in the centre of gravity of each finite element are calculated with relations:

$$
\left\{\begin{array}{l}
v_{z}^{e}=\frac{4}{\alpha_{0} \overline{a_{2}}} A_{N 2} \psi_{N}^{e} \\
v_{r}^{e}=\frac{4}{\alpha_{0} \overline{a_{2}}} A_{N 1} \psi_{N}^{e}
\end{array}\right.
$$

Meridians speed module will be calculated with the relationship:

$$
v_{m}^{e}=\sqrt{\left(v_{z}^{e}\right)^{2}+\left(v_{r}^{e}\right)^{2}}
$$


Speed on borders is calculated by extrapolation. When calculating the pressure a Bernoulli equation is applied along a stream lines, between a point on the inlet border and a current point on the domain, points belonging to the same stream line. If on the boundary $A B$ velocity is constant and equal with $v_{0}$ and pressure $p$ is $p_{0}$ we have the Bernoulli equation:

$$
p=p_{0}+\frac{\rho}{2}\left(v_{0}^{2}-v_{m}^{2}\right)
$$

Dividing the current speeds and pressures with $p_{0}$ and $v_{0}$ so that we form dimensionless calculation:

$$
\begin{gathered}
\overline{v_{m}}=\frac{v_{m}}{v_{0}} \\
\bar{p}=C_{p}=\frac{p-p_{0}}{\rho \frac{v_{0}^{2}}{2}}=1-\left(\overline{v_{m}}\right)^{2}
\end{gathered}
$$

Figures 5 and 6 present the speeds variation in meridian plane along the stream lines, respectively the pressure coefficient $C_{p}$ for the two types of impellers.

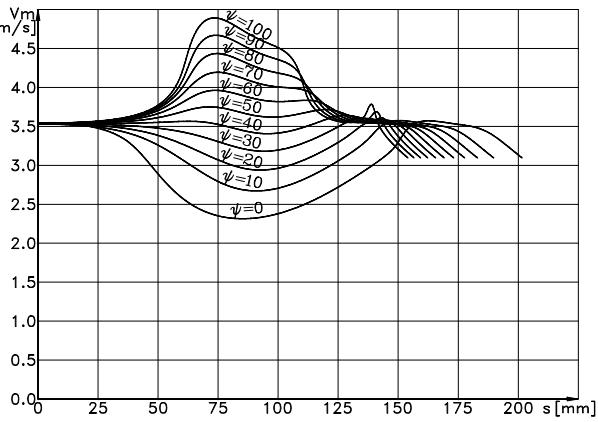

a) $\mathrm{n}_{\mathrm{q}}=25,7$

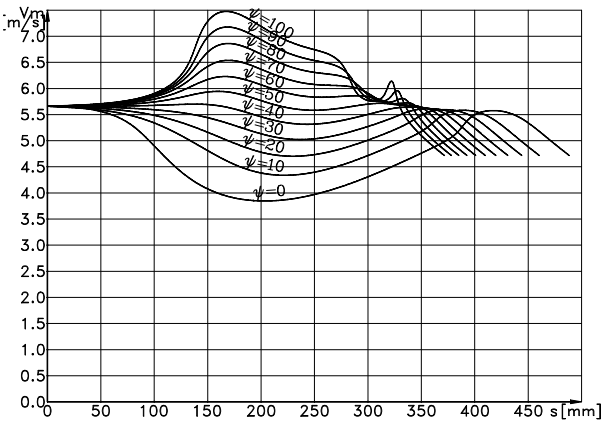

b) $\mathrm{n}_{\mathrm{q}}=80,8$

Fig. 5. Speed variation along stream lines

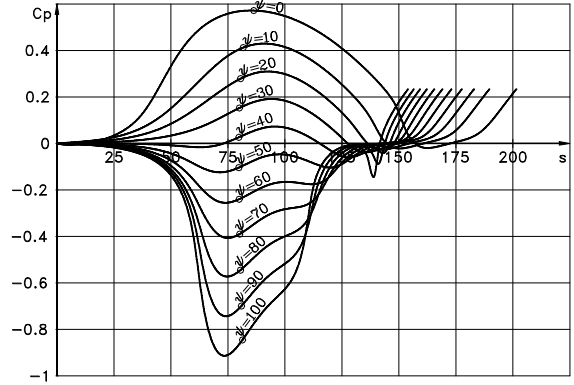

a) $\mathrm{nq}=25,7$

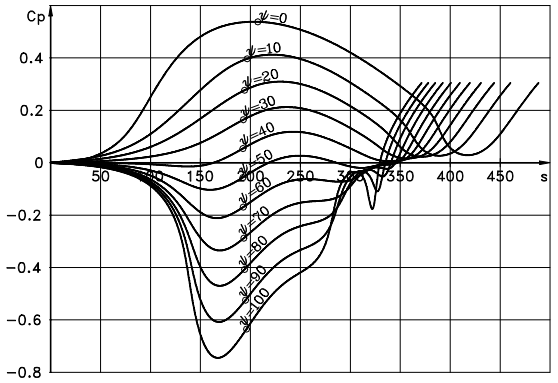

b) $\mathrm{nq}=80,8$

Fig. 6. Pressure coefficient variation along stream lines 


\section{Choosing the blade area in the domain of hydrodynamic field}

Classic calculus relations and design method are combined with facilities of computer use in this phase. Meridian hydrodynamic field data in an optimized domain depending of impeller type offers the perspective of results close to reality. Sections of the calculation are equal to the number of stream lines (11 lines of flow) which means doubling or even tripling them to the case of using the graphic-analytical method of tracing the hydrodynamic field.

Choice of blade area in as many variations or options initially imposed is an additional possibility to optimize the blades.

From the preliminary study it is known where $D_{2}$ will be located on the output edge. In the meridian plan will be a straight with inclined angle $\gamma_{2}$ (Fig. 7). If $\mathrm{n}_{\mathrm{q}} \leq 35$ is recommended that $\gamma_{2}=0$, and for $n_{\mathrm{q}}>35$ is acceptable so that it can tilt somewhat orthogonal to the middle flow line. Approximate statistical relationship can be used, (Gyulai, 1988):

$$
\gamma_{2}=44,037 \lg n_{q}-68,213
$$

The output edge pivots around point $P_{m e}$ at radius $r_{2}=D_{2} / 2$ on the medium stream line. The final decision on the angle $\gamma_{2}$ is taken after reasons that follow.

At the inlet there is an infinitely of possible solutions. Angle is also given guidance by (Gyulai, 1988):

$$
\gamma_{1}=55,19 \lg n_{q}-53,726
$$

With the marked output edge marked image from fig. 7 is obtained; in which on the shroud edge are given the points of the contour mesh. Choose the position of $P_{m i}$ (the pivot edge inlet) so that there is enough space to carry blades because otherwise it will result in a disproportionate number of blades. Because the inlet edge is situated in the curvature of the hydrodynamic field is necessary correlate its position with extreme speed values near the shroud because as meridians are maximum and speed of transport $u_{1}$ is also maximum and at the hub the situation is reversed in the sense of extreme minimum.

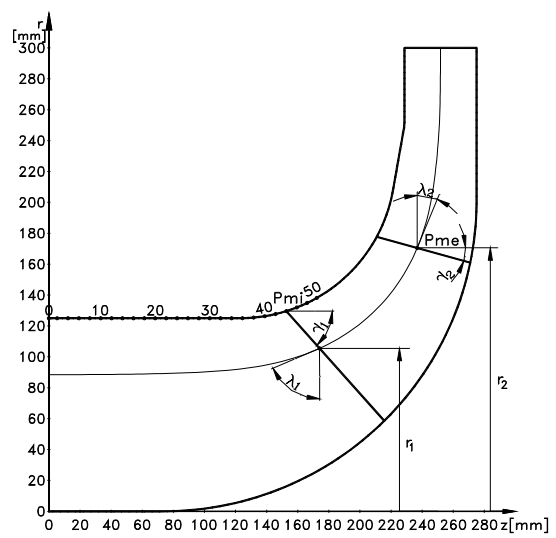

Fig. 7. Marking the inlet and outlet edges 
These two extreme speed values involve the angle $\beta_{1}^{\prime}$ whose values will be slightly different between the shroud and hub. Only the blades zone is retained from the stream lines domain and speeds and pressures. From this moment the rest of the hydrodynamic field doesn't interest us in terms of usefulness for the blade zone, only the edges between input and output. At this stage any option may be followed by a rerun, if the image of velocity variation in the blade (Fig. 8) does not meet the target. Next we are interested only in the hydrodynamic field of the impeller area where we want to avoid areas at risk for increased sensitivity to cavitation or emphasis of the degree of blockage. Therefore after a first option to input the input and output edges, the variation of speeds along the stream lines is displayed again with markings for the blade area (bold in Fig. 8). It is generally better that the input edge is in the extremes of speed curves or after in the flow direction. The justification is given by two aspects:

1. after the stream inlet in the blades zone is good for speeds to have tended to decrease rather than increase, which would increase the risk of cavitation.

2. height of speed triangles from input (Fig. 10) is affected by the values of these speeds and by these the construction blade angle of the inlet, i.e. high levels of transport speed, $u_{1}$, (SL shroud) correspond to proportional values for the $v_{m}$ and vice versa to the hub, so the angle does not fall below $10^{\circ}$.

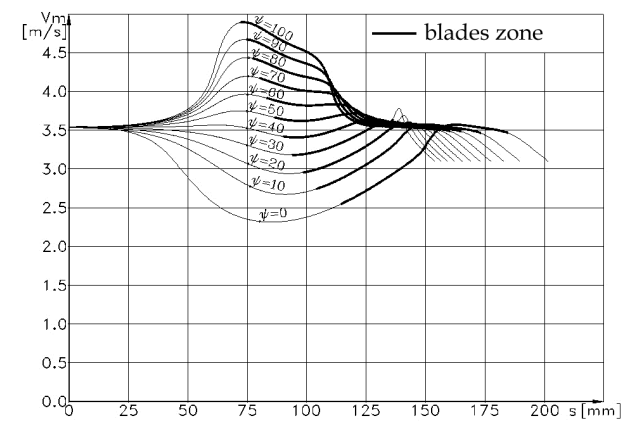

a) $\mathrm{n}_{\mathrm{q}}=25,7$

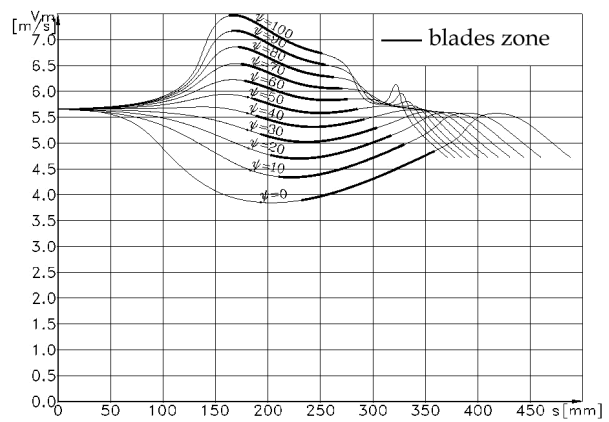

b) $\mathrm{n}_{\mathrm{q}}=80,8$

Fig. 8. Marking changes in velocity in the blade

If the angle falls below $10^{\circ}$ there will be problems with the degree of blockage in an inlet in that $\rho_{1}<0.6$, which is again unacceptable because of increased susceptibility to cavitation.

After the final decision on the position of input and output edges, the meridian velocity variation along of input and output edges is presented. These speeds will be used to calculate kinematic and angular elements of inlet and outlet, which offer height of speed triangle.

\section{Blade inlet design}

Initial sizing data for inlet are meridian speeds on the inlet edge at the intersection points of stream lines with inlet edge (radiuses resulted), blade thickness (minimum $4 \mathrm{~mm}$ ), transport speeds (tangential) at the point of calculation: $u_{1}=r_{1} \omega$. The number of blades is determined by statistical relationship (Pfleiderer, 1961): 


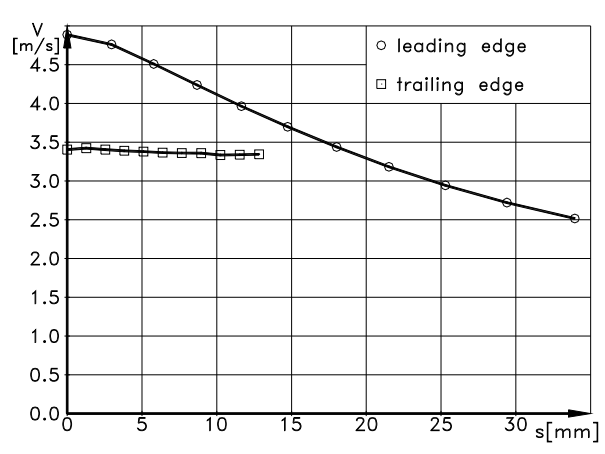

a) $\mathrm{n}_{\mathrm{q}}=25,7$

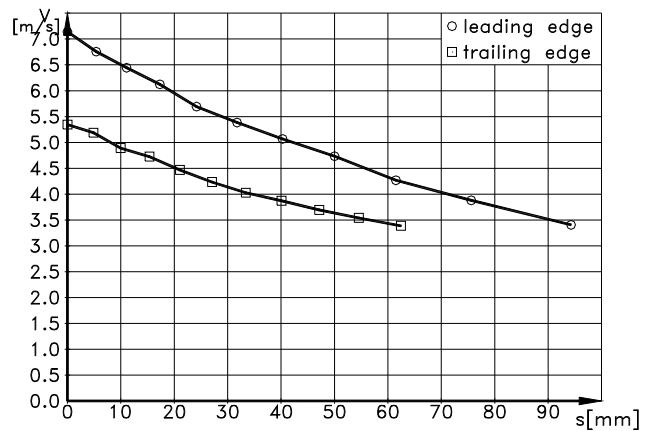

b) $\mathrm{n}_{\mathrm{q}}=80,8$

Fig. 9. Velocity variation along the meridians of input and output edges

$$
z_{1}=4 \pi \sin ^{2} \beta_{m} \cos \lambda_{m} \frac{r_{2}+r_{1}}{r_{2}-r_{1}}
$$

where $\beta_{m}=\frac{\beta_{1}+\beta_{2}}{2}, \lambda_{\mathrm{m}}=\frac{\lambda_{1}+\lambda_{2}}{2} r_{1}, r_{2}, \lambda_{1}, \lambda_{2}$ (fig. 7.) are data related to medium stream line. Angle $\beta_{2}$ is calculated in a first approximation by the statistical relationship, (Gyulai, 1988):

$$
\beta_{2}=\frac{106}{n_{q}^{0,235}}
$$

and $\beta_{1}$ is the angle of inlet without blockage given by the thickness of the blade

$$
\beta_{1}=\operatorname{arctg} \frac{v_{m 1}}{u_{1}}
$$

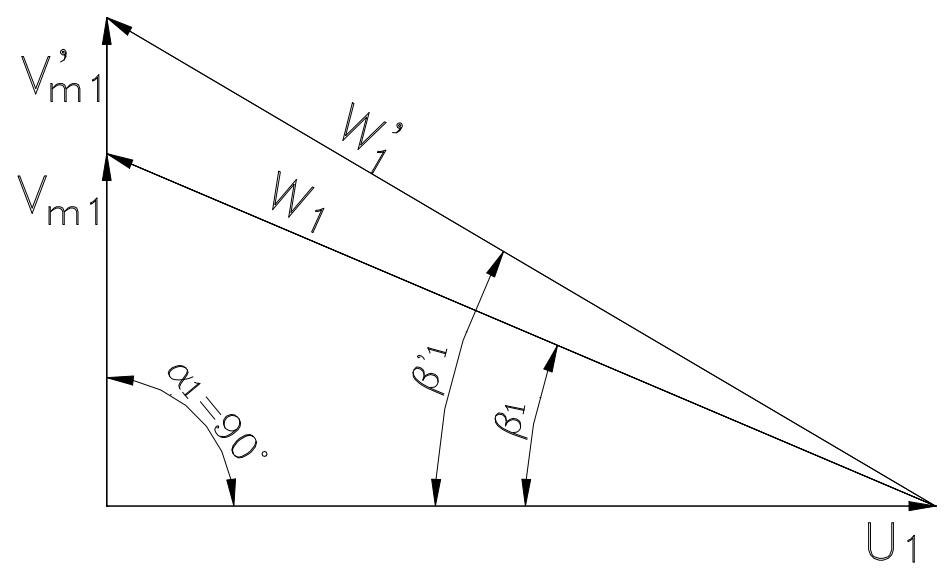

Fig. 10. Velocity triangles at the impeller inlet 


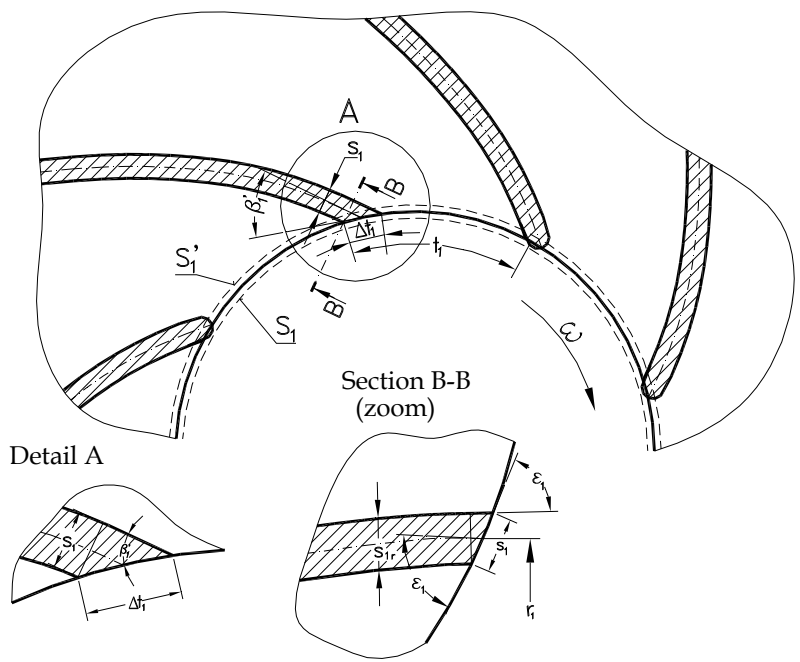

Fig. 11. Blades blockage at inlet

The essential condition for inlet is the alignment of the calculated blade direction to the stream direction given by the inlet speeds triangle. Blades by their inclined position in the impeller channel and their thickness causes a decrease of the normal passing section through the entire blades zone. The measure of this reduction is given by the degree of blockage which in geometric terms is the ratio of the blocked section and unobstructed section. To this effect the degree of blockage, $\rho_{1}$, given the thickness of the blades $s_{1}$, must be taken into account:

$$
\rho_{1}=\frac{S_{1}^{\prime}}{S_{1}}=\frac{b_{1}\left(t_{1}-\Delta t_{1}\right)}{b_{1} t_{1}}=1-\frac{\Delta t_{1}}{t_{1}}=1-\frac{\Delta t_{1}}{\frac{2 \pi r_{1}}{z_{1}}}
$$

In figure 11 is observed that $\Delta t_{1}$ is blade thickness on the inlet section and on the stream surface. Reference thickness, $s_{1}$, based on the hydrodynamic flow through the impeller is thickness measured in a direction normal to the blade contour from the stream surface. If $\beta_{1}^{\prime}$ is blade construction angle of inlet then from the inlet speed triangle can be written as:

$$
\Delta t_{1}=\frac{s_{1}}{\sin \beta_{1}^{\prime}}
$$

Substituting in (27) one gets the calculating relation of the degree of blockage of the fluid depending on the thickness measured on the stream surface:

$$
\rho_{1}=1-\frac{z_{1} s_{1}}{2 \pi r_{1} \sin \beta_{1}^{\prime}}
$$

Real thickness, $s_{1 r}$, of the blade is the thickness measured perpendicular to the blade surface and it counts in the calculation of resistance and the formation of the casting model. It is 
highlighted under section B - B in Fig. 11 and is calculated based on the tilting of the blade surface to the plane tangent to the flow surface at the point of view, tilt angle estimated by $\varepsilon_{1}$, so that:

$$
s_{1 r}=s_{1} \cdot \sin \varepsilon_{1}
$$

The degree of blockage depending on real thickness is calculated by the relationship:

$$
\rho_{1}=1-\frac{z_{1} s_{1 r}}{2 \pi r_{1} \cdot \sin \beta_{1}^{\prime} \cdot \sin \varepsilon_{1}}
$$

Relation (31) resulted in geometric terms. Using a result of the continuity relationship (flow volume) will result the link of the blockage degree with the cinematic elements of inlet, (speeds). Having no ante-stator, normal inlet is considered, $\alpha_{1}=90^{\circ}$, (Fig. 10). Meridian speed with blockage is calculated under the formula:

$$
v_{m 1}^{\prime}=\frac{v_{m 1}}{\rho_{1}}
$$

then the resulting angle of blades construction at inlet:

$$
\beta_{1}^{\prime}=\operatorname{arctg} \frac{v_{m 1}^{\prime}}{u_{1}}=\operatorname{arctg} \frac{\operatorname{tg} \beta_{1}}{\rho_{1}}=\operatorname{arctg} \frac{\operatorname{tg} \beta_{1}}{1-\frac{z_{1} s_{1 r}}{2 \pi r_{1} \cdot \sin \beta_{1}^{\prime} \cdot \sin \varepsilon_{1}}}
$$

If in relation (33) $v_{m 1}^{\prime}$ is replaced with (32) or $\rho_{1}$ with (31) we get a default formula for $\beta_{1}^{\prime}$. Its solution is obtained through an iterative calculation initiated by an approximated value of $\rho_{1}$ $\left(\rho_{1}=0.8\right)$. The resulting array is fast converging to the sought value.

For centrifugal pumps with low or middle $n_{q}$ the thickness of the blades is usually constant between inlet and outlet. Only at the inlet rounding is practiced by a circle or ellipse arc so as to not have one of the cases marked NO in Fig. 12. These conditions favour flow separation at the inlet and thus favour cavitation occurrence.
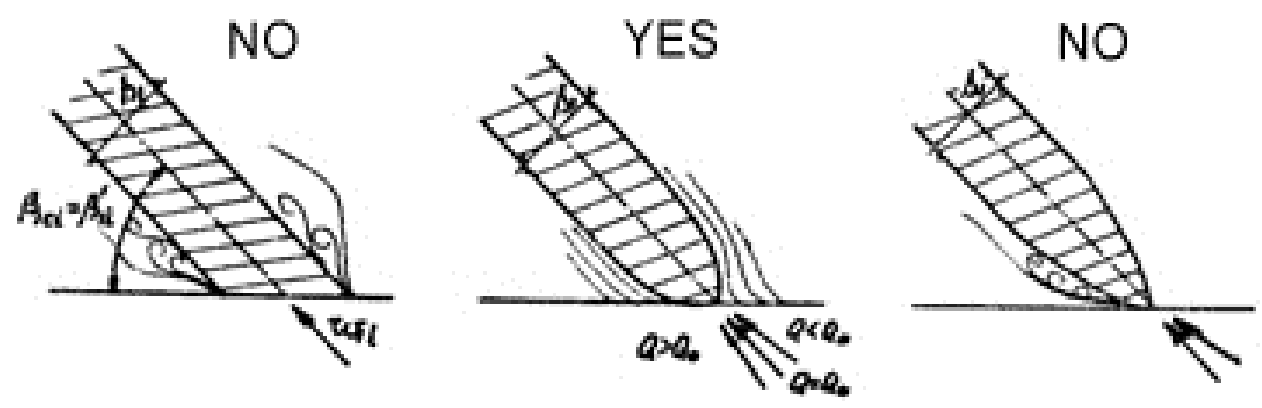

Fig. 12. Blade inlet shape (Gyulai, 1988) 


\section{Blade outlet design}

Initial blade outlet data are meridian speeds in edge points of the outlet edge, radiuses of these points, blade thickness (minimum $4 \mathrm{~mm}$ ), transport speed in such points $u_{2}=\omega r_{2}$. At outlet occurs the deflection effect of the flow from the direction of blade. Quantifying it is the coefficient of Pfleiderer, p (Pfleiderer, 1961):

$$
p=k_{p}\left(1+\frac{\beta_{2}^{[\circ]}}{60}\right) \frac{r_{2}^{2}}{z_{2} S}
$$

where $S=\int_{x_{1}}^{x_{2}} r \mathrm{dx}$ is static moment of the arc-line between inlet and outlet of the streamline. Streamline is given by points and for the numerical calculation of the integral the summation is used:

$$
S=\int_{x_{1}}^{x_{2}} r \mathrm{~d} x \cong \sum_{i=1}^{I_{\max }}\left(\frac{r_{i}+r_{i-1}}{2}\left(x_{i}-x_{i-1}\right)\right)
$$

Fig. 13. Geometric schema for static momentum for one streamline

In relation (35) summation is done on small arcs that approximate the centre of gravity of the bowstring. For coefficient $k_{p}$ values recommended by what is following the impeller in three different cases:

- $\quad k_{p}=1 \ldots 0.85$ for impeller followed by a space without blades

- $\quad k_{p}=0.85 \ldots 0.65$ for impeller followed by collector

- $\quad k_{p}=0.65 \ldots 0.60$ for impeller followed by stator blades

Assuming infinite number of blades: $z_{2} \rightarrow \infty$, flow deviation tends to zero. Thus the outlet will consider two triangles of speeds (Fig. 14), $\Delta_{2}$ corresponding to the geometrical construction of blade, and for the real fluid motion with deflection, triangle $\Delta_{3}$.

Hydraulic moments corresponding to the two situations are in the ratio:

$$
\frac{M_{h \infty}}{M_{h}}=\frac{\rho Q_{t}\left(r_{2} v_{u 2}-r_{1} v_{u 1}\right)}{\rho Q_{t}\left(r_{2} v_{u 3}-r_{1} v_{u 1}\right)}=1+p
$$




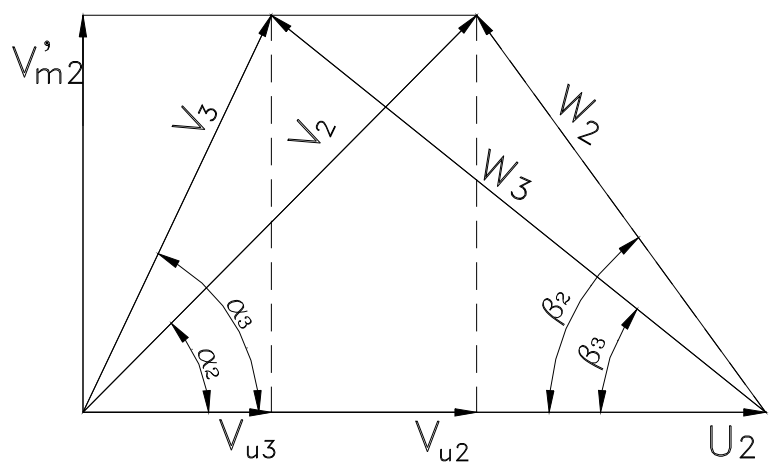

Fig. 14. Velocity triangles at the impeller outlet

Outlet energy transfer depends on three parameters with three degrees of freedom thus:

1. outlet impeller diameter, $\mathrm{D}_{2}$

2. construction angle of blade, $\beta_{2}$

3. number of blades out $\mathrm{z}_{2}$ (implied network density)

Simultaneous optimization of all three options is very difficult. Therefore, in the first phase, for the diameter $D_{2}$ the initially determined value by the head coefficient, $\psi_{\mathrm{H}}$, is accepted and for the other two several possible variants are combined. Number of blades is stored as options calculated initially at inlet. If that number doubles or triples this is taken into account, and outlet options will be double or triple than inlet. For $\beta_{2}$ first is tested the rounded expected value, given by (25), then depending on results are also allocated other possible variants near baseline until achieving the conditions imposed by check, (Gyulai, 1988):

a. achieve of theoretical pumping head, $H_{t}$

b. the condition of optimum solidity $\left(l / t_{m} \cong 2\right)$

Most important relations used in the calculations (except those relating to the results of solving the kinematic and angular elements through solving triangles of speeds) are:

- $\quad$ Theoretical pumping head $H_{t}$ :

$$
H_{t}^{\prime}=\frac{1}{g} u_{2} v_{u 3} \geq H_{t} \quad \text { (from preliminary calculation) }
$$

- $\quad$ Theoretical pumping head assuming infinite number of blades $H_{t \infty}$ :

$$
H_{t \infty}=\frac{1}{g} u_{2} v_{u 2}
$$

- The relationship between the two pumping heads:

$$
\frac{H_{t \infty}}{H_{t}^{\prime}}=\frac{v_{u 2}}{v_{u 3}}=1+p
$$


Outlet calculation is a calculation to check the " $n$ " variants out of which the optimal variant is chosen as the best option that is closest to the initial conditions, i.e. achieving theoretical pumping head and the condition of optimum solidity $(\cong 2)$.

\section{Calculation of the blade surface in the space frame between inlet and outlet using CAD techniques}

The route of inlet to outlet is resolved by interpolating one of the significant kinematic quantities for load distribution along the inter-blades channel of impeller. Interpolation is done along the streamline (flow 3D surface) controlled by curvilinear coordinate " $x$ " and not by the current radius " $r$ " because it better quantifies the load (load distribution) in the radialaxial zone. Hydraulic momentum is the size that reflects blade loading between inlet and outlet. For a current point of curvilinear coordinate $x$ it says:

$$
M_{h x}=\rho Q_{t}\left(r_{x} v_{u x}-r_{1} v_{u_{1}}\right)
$$

From (40) specifying $\left(r v_{\mathrm{u}}\right)_{\mathrm{x}}$ follows:

$$
\left(r v_{u}\right)_{x}=r_{1} v_{u 1}+\frac{M_{h x}}{\rho Q_{t}}=f(x)
$$

Size directly related to hydraulic momentum is $\left(r v_{u}\right)_{x}$, so if the variation of product $r v_{u}$ along impeller channel is controlled, then results the variation of hydraulic momentum versus radius, partial pumping head distribution, distribution of pressure differences on the faces of the blade. $r v_{u}$ product variation implies variation of the construction angle $\beta$ necessary under the assumption that the relative velocity is tangential to the middle surface of the blade. Height of speeds triangles is given by meridian speed, $v_{m}^{\prime}$, which is $v_{m}$ corrected with degree of blockage, $\rho_{1-2}$, resulted from thickness of the blades. If we consider a current point on streamline denoted by "i", then current angle $\beta_{i}$ is resulting from the relationship:

$$
\beta_{i}=\operatorname{arctg}\left(\frac{v_{m i}}{\rho_{i}} \frac{1}{u_{i}-v_{u i}}\right)
$$

It is noted that in (42) appear factors $\mathrm{r}_{\mathrm{i}}\left(u_{i}=r_{i} \omega\right)$ and $\mathrm{v}_{\mathrm{ui}}$, so no matter which of the two variants are interpolated $\left(\mathrm{rv}_{\mathrm{u}}\right.$ or $\left.\beta\right)$, we get the same kind of information. Most often angle $\beta$, is chosen, being directly related to the blade channel orientation in impeller.

$\beta$ angle variation between inlet and outlet must be chosen so that there is a relatively uniform blade loading and the variation is strictly increasing throughout the area. For a better flow engage at the inlet and outlet, as stated above, it is recommended that at neighbourhood extreme points, the blade loading tend to zero. Analyzing several cases of impellers that condition is satisfied if the curve of $\beta$ variation have derived zero at inlet and outlet. Computer solving is possible only through an analytical generation.

\section{$11.1 \beta$ angle interpolation with two connected parabola arcs}

Connecting the two arcs of parabola is the point $x_{3}$ as common tangent (Fig.15). Functions that define two parabola arcs with vertical focal axis noted by $f_{1}$ and $f_{2}$ with general equations, (Milos, 2009): 


$$
\left\{\begin{array}{l}
f_{1}(x)=a_{1} x^{2}+b_{1} x+c_{1} \\
f_{2}(x)=a_{2} x^{2}+b_{2} x+c_{2}
\end{array}\right.
$$

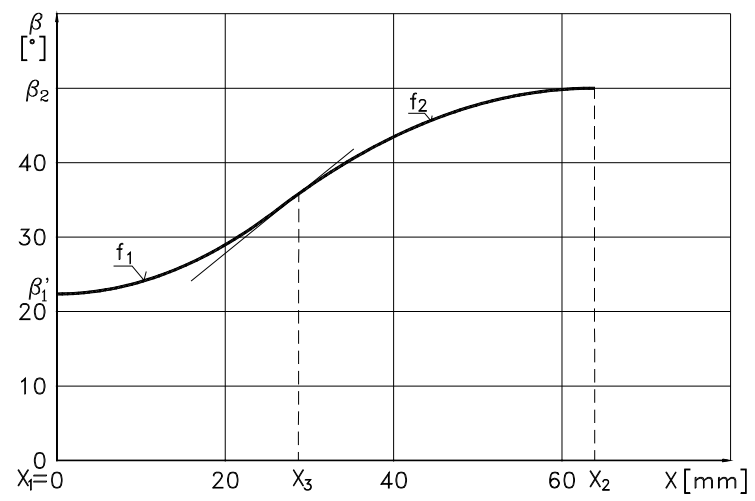

Fig. 15. $\beta$ angle interpolation with two connected parabola arcs

It is noted that in order to concretely define is necessary to know the coefficients $a_{1}, b_{1}, c_{1}, a_{2}$, $b_{2}, c_{2}$. So we need six equations with six unknowns derived from equations (43). Putting analytical positioning and connection conditions we have under notation of Fig. 15:

i. the two parabola arcs are connected by the same tangent in $x_{3}$

ii. the first arc of parabola is tangential to the horizontal (has derivative zero in $x_{1}$ )

iii. the second arc of parabola is tangential to the horizontal (has derivative zero in $x_{2}$ )

iv. the two parabola arcs have the same value in $x_{3}$

v. the first arc of parabola passing through the point coordinates $\left(x_{1}, \beta_{1}^{\prime}\right)$

vi. the second arc of parabola passing through the point coordinates $\left(x_{2}, \beta_{2}\right)$

Translating the six analytical conditions will result in a system of six equations with six unknowns (44) is solved exactly by Gauss elimination algorithm.

$$
\begin{cases}f_{1}^{\prime}\left(x_{3}\right)=f_{2}^{\prime}\left(x_{3}\right) & (\mathrm{I}) \\ f_{1}^{\prime}\left(x_{1}\right)=0 & (\mathrm{II}) \\ f_{2}^{\prime}\left(x_{2}\right)=0 & (\mathrm{III}) \\ f_{1}\left(x_{3}\right)=f_{2}\left(x_{3}\right) & (\mathrm{IV}) \\ f_{1}\left(x_{1}\right)=\beta_{1} & (\mathrm{~V}) \\ f_{2}\left(x_{2}\right)=\beta_{2} & (\mathrm{VI})\end{cases}
$$

Point position $x_{3}$ in interval $\left(x_{1}, x_{2}\right)$ changes step by step until the optimal shape of a blade skeleton variation will result. It is noted that blade load has an infinite range of solutions for each streamline (in 3D surface flow). If each streamline is treated separately charging flow is unlikely to result in a smooth surface (non-sinuous). Blade loading for each streamline must be linked with loads on neighbouring lines. Viewing blade loading by the $\beta$ angle is necessary but not sufficient. Calculation should be continued until the skeleton surface 
projection of the blade in a plane perpendicular to the axis of rotation is obtained and possibly even 3D surface representation of the vane. Numerical values obtained and views these images provide enough information to see trends and take appropriate corrective decisions for load range.

This impeller design phase involves many calculations and graphics based on large amounts of data. Optimum solution is possible using a structured computer program.

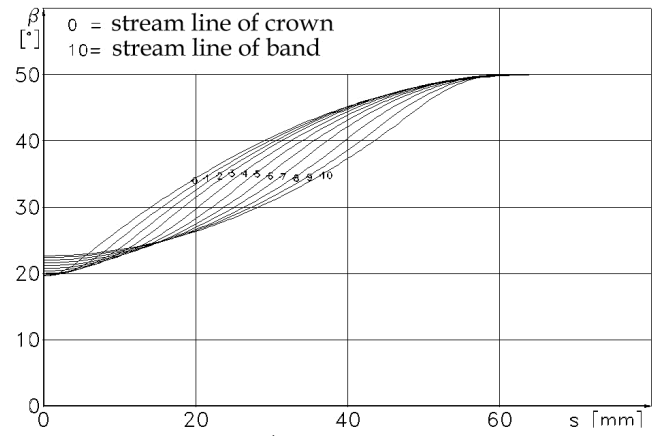

a) $\mathrm{n}_{\mathrm{q}}=25,7$

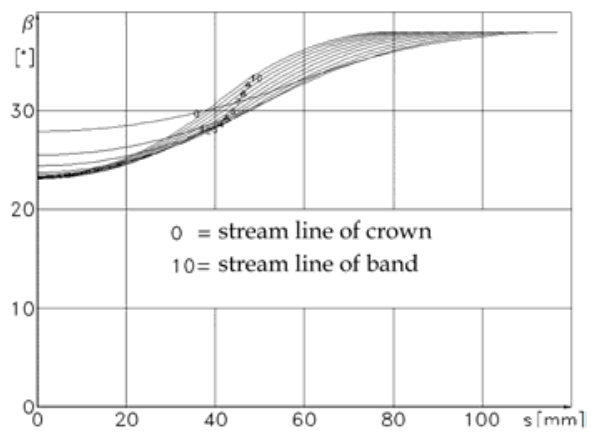

b) $\mathrm{n}_{\mathrm{q}}=80,8$

Fig. 16. Variation of $\beta$ angle optimized for the two impeller types, (Milos, 2009)

\subsection{Skeleton surface of the blade in projection on a plane perpendicular to the axis of rotation}

Design studies and calculations of the impeller must be completed with an execution drawing. From the set up at this stage, the impeller blade contour was obtained in meridian plan. Starting from this design and adding some thick walls on the perimeter of front and rear shroud, followed by setting up the hub and impeller mounting area on the shaft, a drawing is obtained as a section through the impeller intersected by a plane passing through the rotation axis. Since the section is a piece generated by rotating, other representations are obtained easily in view. What remains not yet clearly defined are the 3D blades shape. The first step in this direction is to obtain the projection surface of the blade in a plane perpendicular to the axis of rotation.

In Figure 17, left, arc 1-2 is a streamline from the meridian plane. On this streamline at the current curvilinear coordinate $x$ is considered discrete portion $\Delta x$. For this portion the projection image on a plane perpendicular to the axis of rotation is obtained. Streamline 1-2, when rotated around the axis of rotation generates surface flow on which moves the fluid particle driven by impeller blade. To highlight the trajectory that the blade must induce on a fluid particle on the strip surface flow generated by the arc $\Delta x$, is preferable that the strip is brought in the plane by developable surface. But the development of a curved surface geometry does not provide the necessary geometric links, so it is recourse to the projection of the strip surface flow on a conical surface, coaxial with the surface flow, which passes through the arc ends $\Delta x$. The error committed by the projection is relatively small given of the small size of the arc $\Delta x$. Basically bowstring $\Delta x$ generates the conical surface in question. Developing the conical surface is performed immediately by arcs with the centre at cone top. 


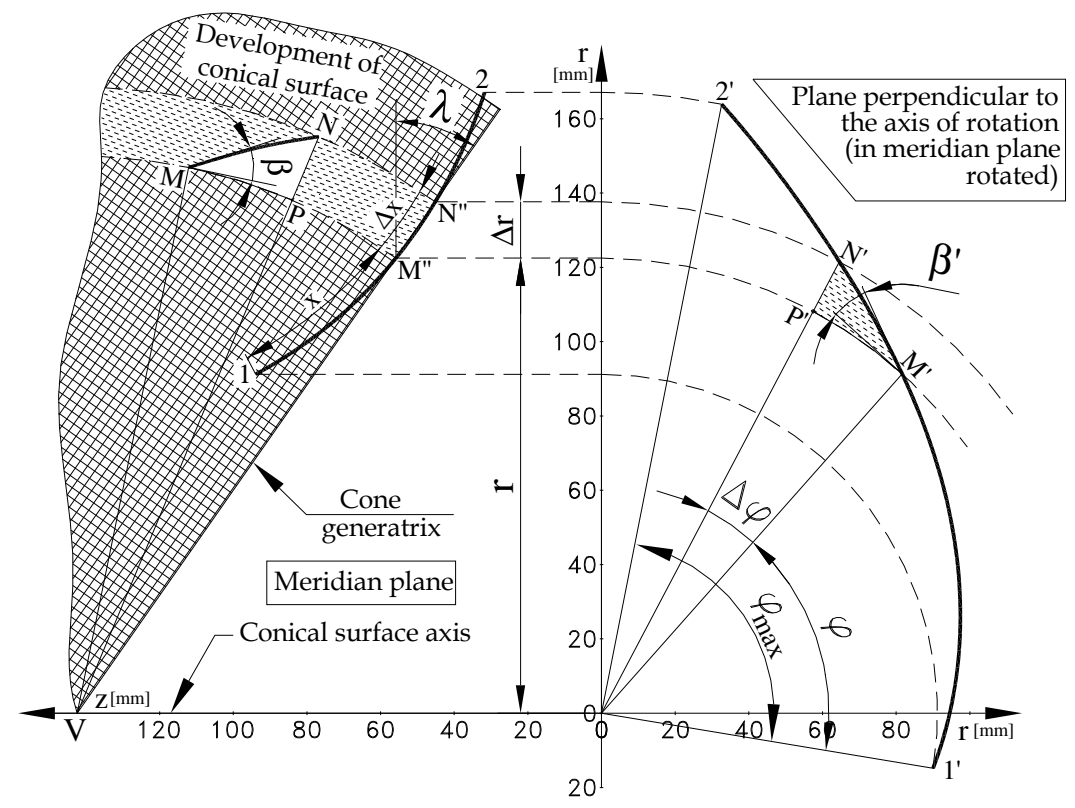

Fig. 17. Transposition route in-out from the stream surface on a plane perpendicular to the axis of rotation, (Milos, 2009)

Now on the strip a fluid particle trajectory can be traced by choosing a start point $M$ and leading the segment $M N$ at angle $\beta$ against the tangent in $M$ to the circle of the developed cone. The angle $\beta$ corresponds to the average area of the chart $\Delta x$ previously generated $\beta=$ $f(x)$ and ultimately reflected in the average load on the blade portion $\Delta x$. Taking radius $N V$ point $P$ is obtained and the triangle $M N P$, with approximation, can be considered to be rectangular. Thus a sequence of fluid particle trajectory is obtained, needed to be done only if this route will provide a solid wall of the impeller blade.

Next is donne the transposition of this portion $\Delta x$ from the meridian plane, on the flow surface, from developing the strip on the flow surface (from all three) in projection on a plane perpendicular to the axis of rotation. The plane in Figure 17 is the plane that was originally is perpendicular to rotation axis $\mathrm{Oz}$, and then was rotated by $90^{\circ}$ in the figure plane. This plane is in polar representation. The vertical axis is the axis Or crossing the meridian plan for this plane. Points 1 and 2 (beginning and end of the streamline of the blade) will be somewhere on the arcs of the same radius from the projection plane. Arc $\Delta x$ in the projection will be between arcs of radius $r$ and $r+\Delta r$. Let $\varphi$ be polar angle measured from some reference straight. Blade portion $\Delta x$ of the projection will appears under $\Delta \varphi$ angle. The following relations can be written:

a. in developed conical surface passing through the ends of the arc $\Delta x$

$$
\operatorname{tg} \beta=\frac{N P}{M P}, \quad N P=\Delta x
$$


b. in the projection plane perpendicular to the axis of rotation

$$
\operatorname{tg} \beta^{\prime}=\frac{N^{\prime} P^{\prime}}{M^{\prime} P^{\prime}}=\frac{\Delta r}{r \Delta \varphi}, \quad N^{\prime} P^{\prime}=\Delta r
$$

Arc $M P$ is projected in real size, becoming $M^{\prime} P^{\prime}$, i.e.

$$
M P=M^{\prime} P^{\prime}=r \Delta \varphi
$$

Replacing the first two relations we have:

$$
\begin{gathered}
\operatorname{tg} \beta=\frac{\Delta x}{r \Delta \varphi} \\
\operatorname{tg} \beta^{\prime}=\operatorname{tg} \beta \frac{\Delta r}{\Delta x}=\operatorname{tg} \beta \cdot \cos \lambda
\end{gathered}
$$

If you go to the limit with small infinites: $\Delta x \rightarrow d x$ and $\Delta \varphi \rightarrow d \varphi$ resulting differential equation:

$$
\operatorname{tg} \beta=\frac{d x}{r \cdot d \varphi}
$$

Separating variables and integrating

$$
\varphi=\int_{x_{1}}^{x} \frac{d x}{r \cdot \operatorname{tg} \beta}
$$

Integration is done along the streamline. The image in projection on a plane perpendicular to the axis of rotation is calculated and represents in the polar coordinates $(r, \varphi), \varphi$ resulting in radians from the relation (51). To solve analytically the integral (51) there should be an analytical dependence $r=r(x)$ and $\operatorname{tg} \beta=f(x)$. Since often discrete values of these dependencies are available, a numerical integration method is preferred. Integration is done by adding the number of partial areas using trapezoids (Fig. 12).

$$
\Delta \varphi_{i}=\left[\left(\frac{1}{r \cdot \operatorname{tg} \beta}\right)_{i}+\left(\frac{1}{r \cdot \operatorname{tg} \beta}\right)_{i+1}\right] \frac{x_{i+1}-x_{i}}{2}
$$

$\Delta \varphi_{i}$ angles calculated with (52) result in radians. Adding them step by step, current wrapping angles of the middle surface of blade are obtained.

$$
\varphi_{i}=\sum_{i=1}^{i} \Delta \varphi_{i}
$$

Thus each point $\left(r_{i}, z_{i}\right)$ of the streamline is associated with an angle $\varphi_{\mathrm{i}}$ and results the defining of the middle surface of the blade in cylindrical coordinates $\left(r_{i}, \varphi_{\mathrm{i}}, z_{i}\right)$

From hydrodynamic field calculations we have $3 \ldots 11$ or even more streamlines. Applying relation (51) for each streamline will result $\varphi_{\max }$. With $\varphi_{\max }$ you can start calculating $\varphi$ 
optimization which seeks to obtain all streamlines wrapping up of the same angle range. Choosing as reference $\varphi_{\max }$ max angle $\varphi$ corresponding streamline medium (line 5 in case we have 11 streamlines) using a special algorithm changes the position of points $x_{3}$, position number between 1 and 99, until maximum $\varphi$ is the same for each streamline. For the impellers with mixed-flow shape this may not be achieved. In this case it is necessary be provided that the differences between $\varphi_{\max }$ are uniformly increasing or decreasing.

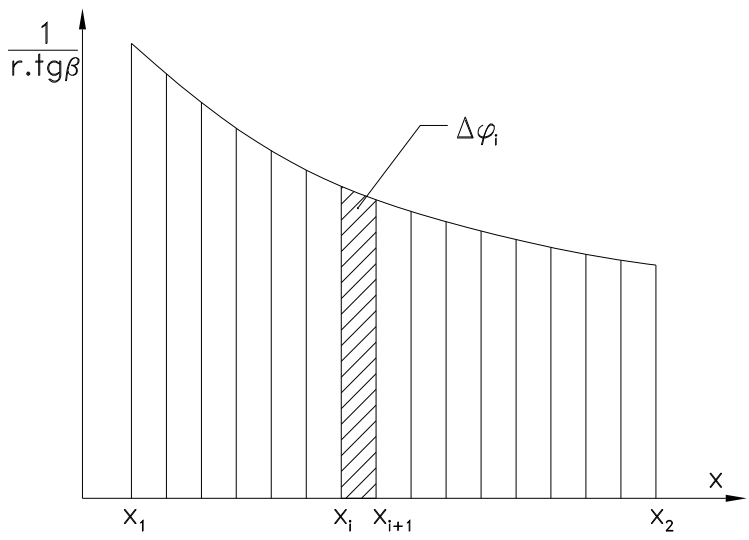

Fig. 18. Numerical integration of the function of wrapping angle of the blade

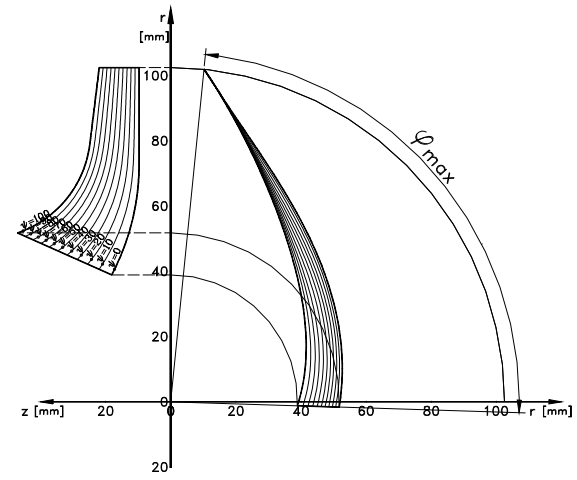

a) $n_{q}=25,7$

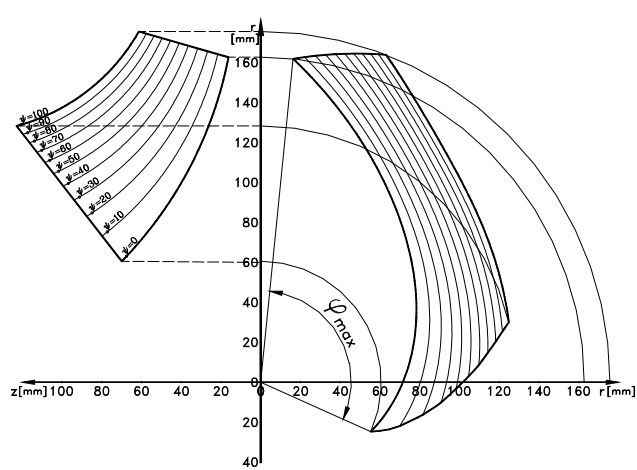

b) $\mathrm{n}_{\mathrm{q}}=80,8$

Fig. 19. Impeller blade in the meridian plane and in the projection plane, a plane perpendicular to the axis of rotation, (Milos, 2009)

With the interpolated angle $\beta$ the middle blades projections in a plane perpendicular to the axis of rotation are calculated. The results are presented in Fig. 19. Figure 20 is presented as an example the projections of all impeller blades for the two distinct types of impellers with small $n_{q}$ (radial) and large $n_{q}$ (mixed-flow). 


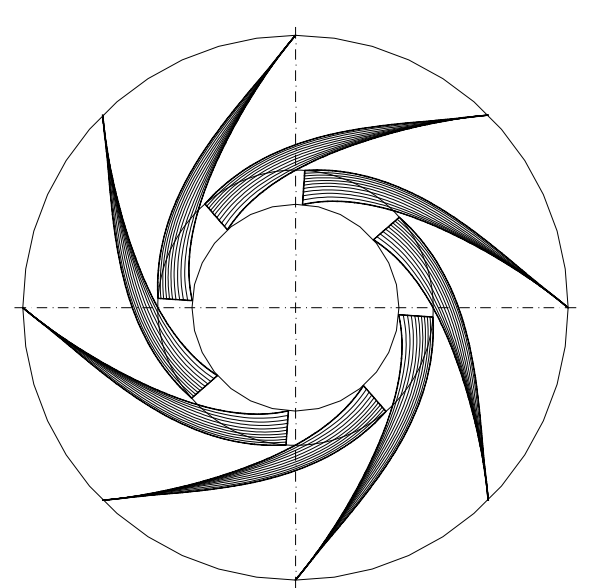

a) $\mathrm{n}_{\mathrm{q}}=25,7$

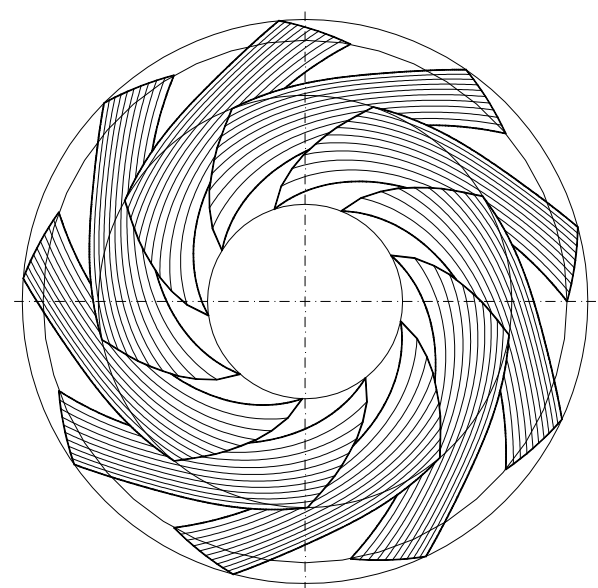

b) $\mathrm{n}_{\mathrm{q}}=80,8$

Fig. 20. Impeller blades in a plane perpendicular to the axis of rotation, (Milos, 2009)

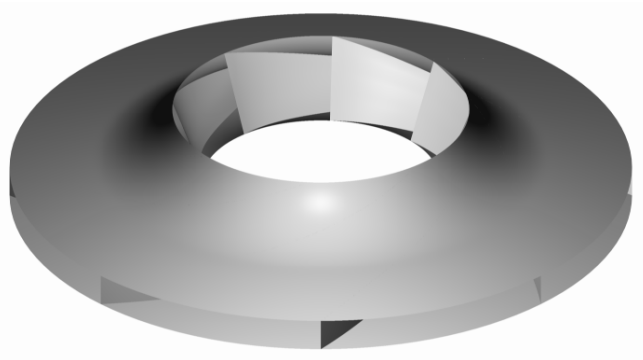

a) $\mathrm{n}_{\mathrm{q}}=25,7$

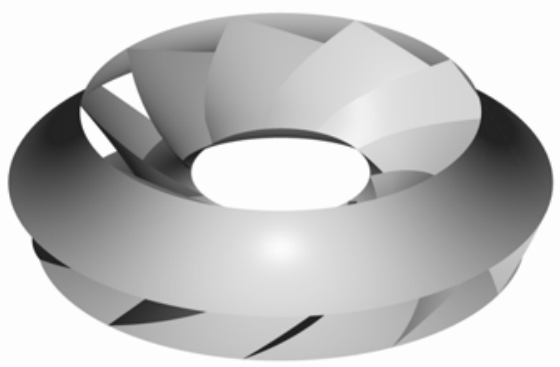

b) $\mathrm{n}_{\mathrm{q}}=80,8$

Fig. 21. 3D view of the impeller (only skeleton surfaces), (Milos, 2009)

\section{Calculus of the skeleton blade surface in the space frame between inlet and outlet using conformal mapping method and CAD techniques}

Regardless of the method of calculating the middle surface of the blade, the initial elements of construction are the angles of inlet, $\beta_{1}$ and outlet, $\beta_{2}$. Construction blade angles between inlet and outlet will be between these limits having a continuous and uniform variation on this course. In terms of geometric transformations Conformal Mapping Method (CMM) (Stepanoff, 1957) means flattening the trajectory on multiple conical surfaces, and in mathematical terms sets a bi-univocal correspondence between the middle curve of the blade on the stream surface and image plane of conformal transformation. Analytical transposition of this method is possible if we observe the conditions that must be fulfilled by function $A(x)$ from the image plane of conformal mapping (Fig. 23) correlated with the streamline of the meridian plane (Fig. 22). What this method adds to the classical ones is thickness accurate transposition versus the middle surface which is also rigorously calculated. 


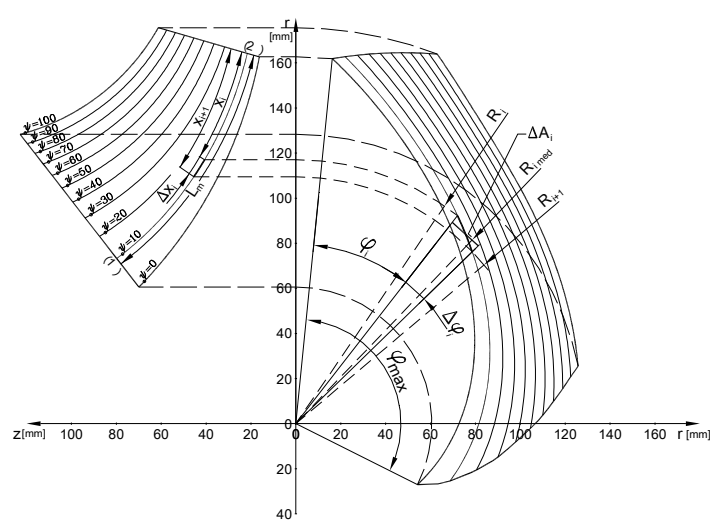

Fig. 22. Correspondence between discrete elements of the blade skeleton in the meridian plane and in projection onto a plane perpendicular to the axis of rotation

These conditions are expressed mathematically:

1. passing through point $O: A(0)=0$;

2. passing through point $M: A\left(L_{m}\right)=A_{M}$;

3. angle of tangent to $A(x)$ in $O$ to be identically with $\beta_{1}:\left.\frac{d A(x)}{d x}\right|_{O}=\operatorname{tg} \beta_{1}$.

4. angle of tangent to $A(x)$ in $M$ to be identically with $\beta_{2}:\left.\frac{d A(x)}{d x}\right|_{M}=\operatorname{tg} \beta_{2}$;

Function which, as allure, is closest to the graphic-analytical solution is a third degree polynomial with four coefficients resulting from resolving the system of equations that is derived from applying the four conditions above.

Function $A(x)=f(x)$ polynomial of degree III, which defines the loading range between inlet and outlet and hence the middle surface geometry is:

$$
A(x)=a x^{3}+b x^{2}+c x+d
$$

Translating analytical the four conditions will result in a system of four equations with four unknowns (55) that solves exactly with Gauss elimination algorithm.

$$
\left\{\begin{array}{l}
A\left(x_{O}\right)=0 \\
A\left(x_{M}\right)=A_{M} \\
A^{\prime}\left(x_{O}\right)=\operatorname{tg} \beta_{1} \\
A^{\prime}\left(x_{M}\right)=\operatorname{tg} \beta_{2}
\end{array}\right.
$$

In Fig. 24 is graphically presented $A(x)=f(x)$ for the 11 streamlines of the meridian plane.

Basically, strips carried on the conical surface (Fig. 17) are here put together in the image plane. Function $A(x)$ must be at least level 3 to allow a second order derivative and thus be able to control the concavity on the work domain which must be uniformly increasing. 


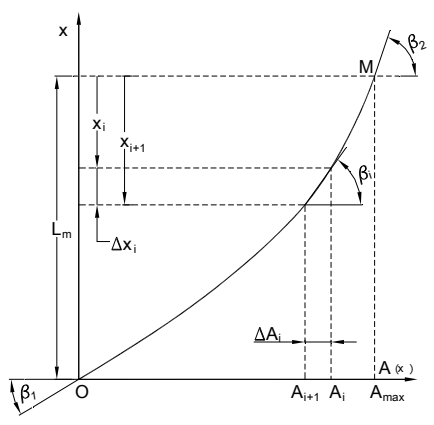

Fig. 23. Image plane of conformal mapping

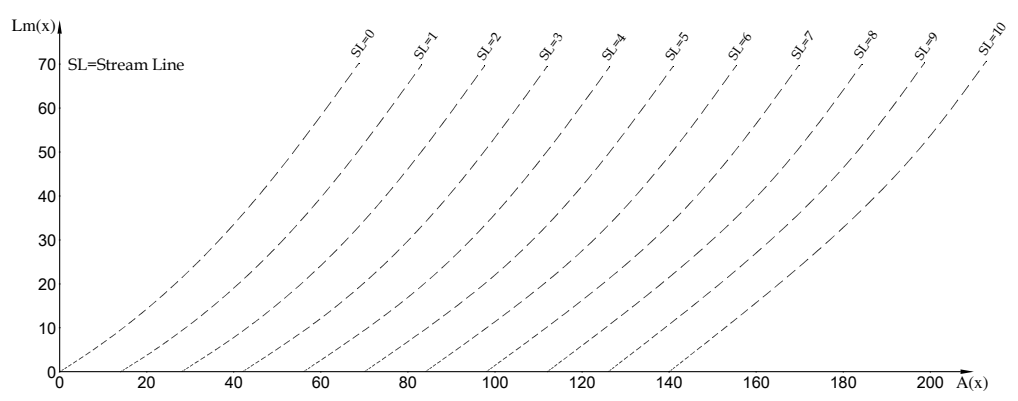

Fig. 24. Functions $A(x)=f(x)$ in a plane of conformal mapping for the 11 streamlines.

According to Figure 23 we see that the blade construction angle $\beta_{i}$ results from:

$$
\operatorname{tg} \beta_{i}=\frac{\Delta x_{i}}{\Delta A_{i}}=\frac{x_{i+1}-x_{i}}{A_{i+1}-A_{i}}
$$

According to notations in Fig. 22, elementary wrapping angle $\Delta \varphi_{i}$ of the projected blade result from the relationship:

$$
\Delta \varphi_{i}=\frac{\Delta A_{i}}{\frac{R_{i}+R_{i+1}}{2}}=\frac{A_{i+1}-A_{i}}{\frac{R_{i}+R_{i+1}}{2}}
$$

Current wrapping angle results from summation of elementary angles as calculated until the current angle by relationship:

$$
\varphi_{i}=\sum \Delta \varphi_{i}
$$

Using a special algorithm the same maximum wrapping angle of each streamline and desired maximum angle can be achieved so that the function $A(x)$ has no inflection and network profiles used in the next phase to be simple curved profiles. Difficulties in obtaining the same $\varphi_{\max }$ for all streamlines and their resolution are similar to those of the previous paragraph. 
Once the middle surface of the blade is obtained, the next step is "dressing" this area with two adjacent surfaces that materializes every half-thickness of the blade. In the case of centrifugal pumps with small and medium $n_{q}$ stream surface sections profiling is practiced with profiles of constant thickness and very low thickness (minimum $4 \mathrm{~mm}$ ). Minimum thickness resulted from traditional technological conditions. If manufacturing technology allows and also the mechanical strength requirements are lower thickness can be lower when appropriate. The only adjustment is made at the inlet of the leading edge where rounding is practiced by a circle arc or ellipse arc, i.e. a semicircle or a semi-ellipse.

For the large and very large $n_{q}$, profiling is appropriate with shapes or profiles of least resistance to fluid movement. These can be obtained from catalogues or user-created profiles using the procedures known in hydrodynamics network profiles. In all cases (including constant thickness) only the thickness function applied to camber line arising from the CMM is used.

\subsection{The transposition of constant thickness in the plane of conformal mapping (Milos, 2009)}

Support curve is the streamline curve of conformal mapping plane. In a current point on the streamline curve of the conformal mapping plane having coordinates $\left(A_{i}, L_{m i}\right)$ current angle $\beta_{\mathrm{i}}$ is already known. According to the schematic representation in Figure 25 the thickness range transposition relations in conformal mapping plane can be written.

- for suction side

$$
\left\{\begin{array}{l}
A_{e x i}=A_{i}+\frac{s}{2} \sin \beta_{i} \\
L_{e x i}=L m_{i}-\frac{s}{2} \cos \beta_{i}
\end{array}\right.
$$

- $\quad$ for pressure side

$$
\left\{\begin{array}{l}
A_{\text {ini }}=A_{i}-\frac{s}{2} \cos \beta_{i} \\
L_{i n i}=L m_{i}+\frac{s}{2} \sin \beta_{i}
\end{array}\right.
$$

At inlet (leading edge) to avoid adverse effects on cavitation given by direct impacts of the fluid flow on a rough form, underside is connected with upside (or vice versa) by an arc of ellipse, Fig. 26. Given that this curve (ellipse) is treated in analytic geometry, those notations will be used.

If $a$ and $b$ are the semi-axes of the ellipse and $s$ is the profile thickness of the leading edge, it appears that we always have $b=s / 2$. Introducing the coefficient, $k_{e}=a / b$ it becomes a control parameter of the connection to leading edge. What must be determined in the first phase are discrete coordinates on the elliptical arc in relation to the local coordinate system $x^{\prime} O^{\prime} y^{\prime}$. These in turn are determined by the mesh streamline curve (support) of the conformal mapping plan. These relations are: 


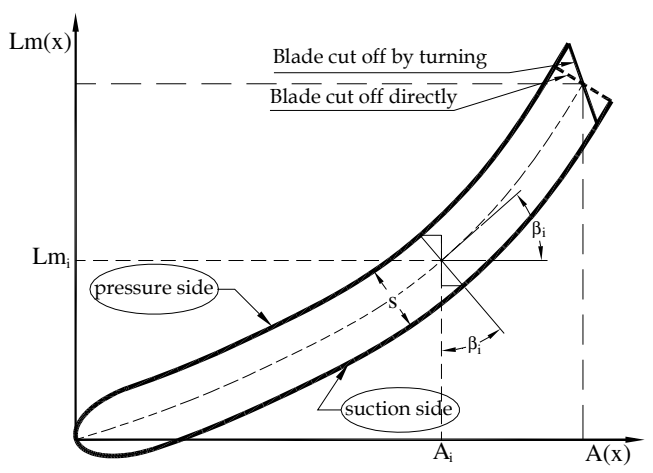

Fig. 25. Schematic representation of the thickness transposition in conformal mapping plan

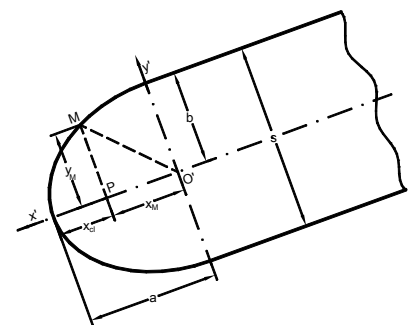

Fig. 26. Leading edge sides connected with an arc of ellipse

$$
\left\{\begin{array}{l}
x_{M}=k_{e} \frac{s}{2}-x_{c l} \\
y_{M}= \pm \frac{1}{k_{e}} \sqrt{\left(k_{e} \frac{s}{2}\right)^{2}-x_{M}}
\end{array}\right.
$$

In subsequent calculations only enters $y_{M}$ (with positive sign) which is semi-thickness with a positive sign and is put in the place of $s / 2$ (59) and (60). For a good outline, especially in the front, it is necessary for a very fine mesh on the portion $a$. On the trailing edge the blade may be cut off straight or cut off by lathing without further processing. In the latter case we identify the extreme points.

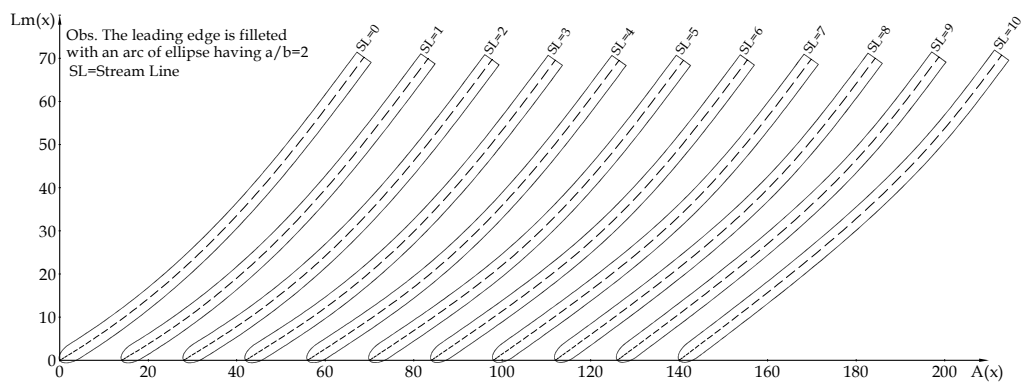

Fig. 27. Thickness transposition in conformal mapping plan for the 11 streamlines 
For example shown in Fig. 27, results for a radial impeller thickness transposition studied in this respect.

\subsection{The transposition of variable thickness in the plane of conformal mapping (Milos, 2009)}

In this case most often are used known section thickness functions, preferably those for which an analytically given function of thickness is known. Support curve is the streamline curve of conformal mapping plane. In a current point on the curve of conformal mapping plane having coordinates $\left(A_{i}, L_{m i}\right)$ angle $\beta_{\mathrm{i}}$ is already known. According to the schematic representation in Figure 28 the same blade thickness relations are used in terms of implementing the transformation line drawn from relations (59) and (60). Semi-variable thickness is calculated with a relation given or inferred.

For example in the case of NACA profiles the following relationship is used:

$$
\frac{y_{d}}{l}=\frac{d}{l} \cdot\left[1,4845 \cdot \sqrt{\frac{x}{l}}-0,63 \cdot \frac{x}{l}-1,758 \cdot\left(\frac{x}{l}\right)^{2}+1,4215 \cdot\left(\frac{x}{l}\right)^{3}-0,575 \cdot\left(\frac{x}{l}\right)^{4}\right]
$$

For calculations $y_{d}$ is semi-thickness, and is put in the place of $s / 2$ from relations (59) and (60). It must be noted that $d$ in (62) is the maximum thickness of the profile and is required by the user. It may be constant or variable with average radius that is the flow surface, depending of resistance conditions imposed to mechanical strain or stiffness, vibration, etc. Using the results of thickness from 4-digit NACA profiles are presented as an example in Fig. 29.

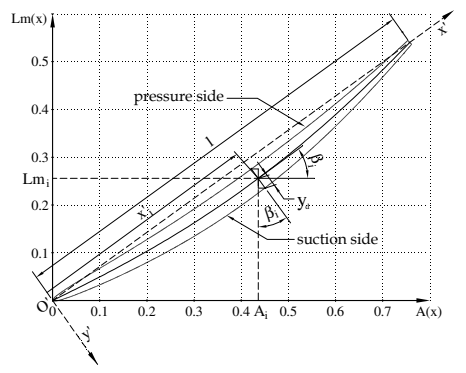

Fig. 28. Schematic representation of the variable thickness transposition of the profile in conformal mapping plane

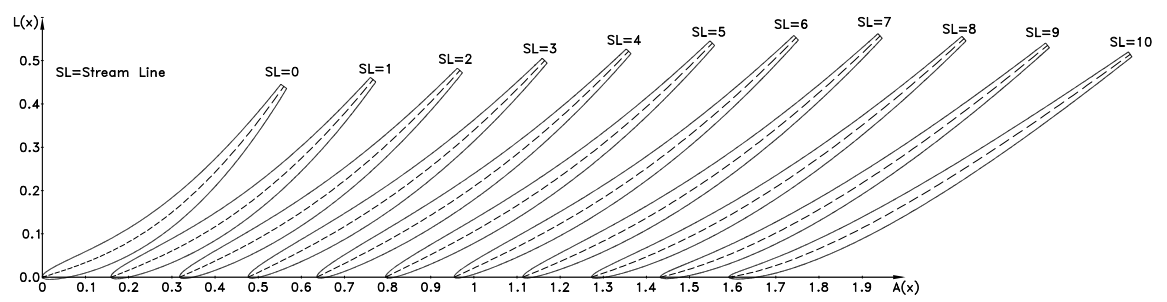

Fig. 29. Transposition of variable thickness in conformal mapping plane using 4-digit NACA profiles for the 11 streamlines 


\subsection{Determination of pressure surface and suction surface of the blade using the transposition of thickness in the plane of conformal mapping (Milos, 2009)}

The process is similar to that of determining the middle surface of the blade except that in this case using a series of already known results for this surface. Having already solved the middle surface of the blade for any of the streamlines, and in conformal mapping planes having implemented thickness profiles (constant or variable) all the prerequisites are there for finding points and support curves of pressure and suction side surfaces. Start is made in conformal mapping plane where for a calculated point on the curve of the middle line of the blade, $A_{s c}$, is immediately founded $L_{i n}$ and $L_{e x}$, see route in Figure 30. Remember that values on axis $\operatorname{Lm}(x)$ are the meridian streamline implementation on this axis in the plane of conformal mapping.

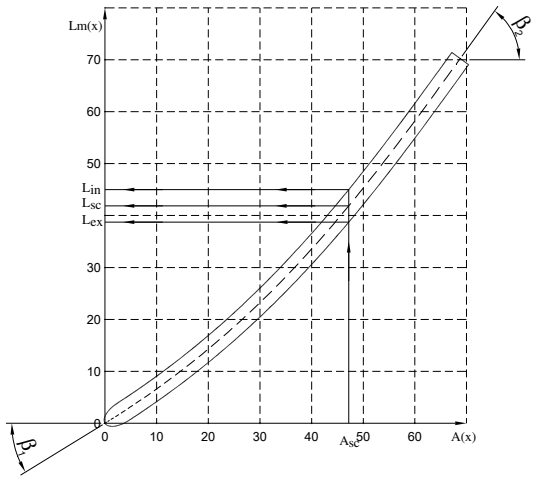

Fig. 30. Identification scheme in conformal mapping plane of the points from pressure surface and suction surface having the same wrapping angle $\varphi$ in projection plane of the blade

The next step is to find by interpolation the position of these points on the streamline belonging to the meridian plane. Finding them depends on how you scroll the streamline. In the case of the optimization phase covering the camber line was recommended to go from outlet to the inlet. Now it is recommended covering to go from the inlet to the outlet, requiring a fine mesh at the leading edge, whether it is constant or variable thickness.

Points once found as positions along meridian plane, involves knowledge of pairs of coordinates $(z, r)$. Passage in the projection plane (perpendicular to the axis of rotation) is made at the corresponding radiuses, followed by polar angle $\varphi_{S C}$ (pole is the point of projection of the axis of rotation on this plane). Size $A_{s c}$ contains, according to relation (57), the wrapping angle of middle line of blade, i.e. each $A_{i}$ is assigned a $\varphi_{\mathrm{i}}$. So as shown in Fig. 29 , three points on the pressure surface, middle surface and suction surface are situated on the same polar straight at identical polar angle, $\varphi_{\text {sc }}$.

Here the three projection curves were represented schematically belonging to three $3 \mathrm{D}$ surfaces: pressure surface, middle surface and suction surface. In the cylindrical projection of the meridian plane they overlap. Resolving requires separate treatment for all their available streamlines. Adding the third coordinate $\varphi$, to the first two $(z, r)$, completes the trio $(z, r, \varphi)$, which is a representation in cylindrical coordinates. Based on the number of 
streamlines and their mesh representation results the smoothness of the surfaces determined in this way.

Note that the thickness of the profiles introduced by this method is the thickness measured on the surface flow. Real thickness (measured in the direction normal to local blade surface) is less or may be at most equal to that introduced in the conformal mapping plane. Therefore the strength and vibration calculations must take this into account.

With the given information the entire impeller and impeller form can be translated on a technical drawing. With the image from the meridian plane the impeller section and the impeller view are constructed. Projection on a plane perpendicular to the axis of rotation is only useful for the blade, which being of a more specific form, curved and twisted in space requires special methods of approach and drawing to be feasible technologically. The design itself consists of several designs resulted from blade cutting with two flat beams.

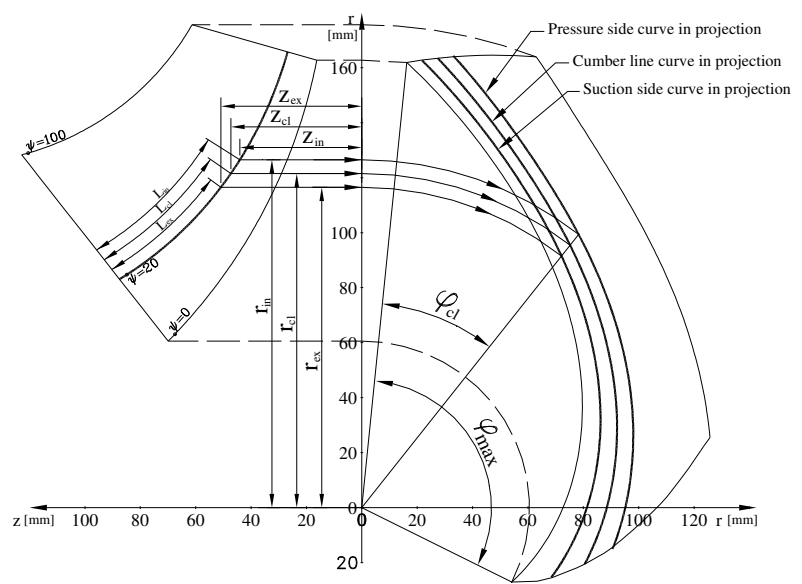

Fig. 31. Identification scheme, in meridian plane of the points from the pressure and suction surface followed by their projection in the plane perpendicular to the axis of rotation

The transition from cylindrical coordinate system in 3D Cartesian coordinate system is through relations:

$$
\left\{\begin{array}{l}
x=r \cdot \cos \varphi \\
y=r \cdot \sin \varphi \\
z=z
\end{array}\right.
$$

In the case of impellers with high $\mathrm{n}_{\mathrm{q}}$, using this method, it is difficult to obtain a uniform blade surface which is generated of the curves determined by the conformal mapping transformation of each stream surface. This operation is done step by step, adjusting blade loading through angle $\beta$. Finally, if the wrapping angle range is not the same for every streamline or is not uniform ascending, the smoothing procedure is repeated.

$3 \mathrm{D}$ representation of the results for visual examination is made in a CAD program with opportunities to read data from data files and their generation with smooth surfaces 
between the mesh networks. Fig. 32 presents a picture of a radial impeller obtained by CMM.

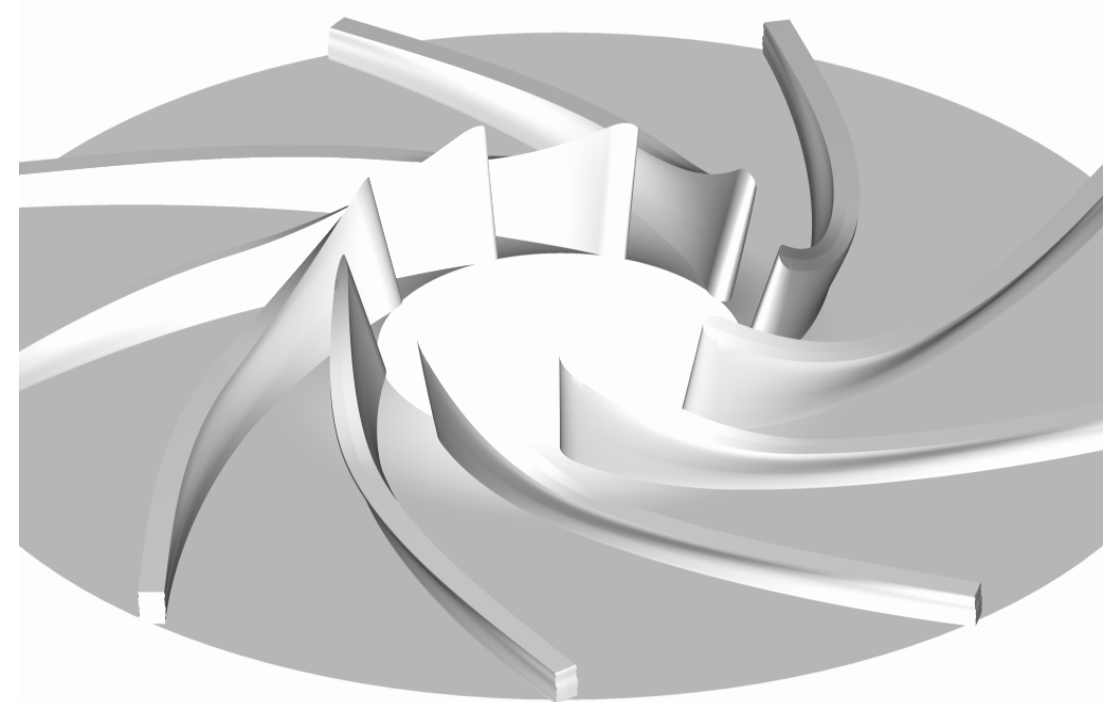

Fig. 32. 3D image of the rear shroud with blades (front shroud disk was removed)

\section{Conclusion}

Using these interpolation methods of $\beta$ angle between inlet and outlet, optimized forms of the centrifugal pump blades can be obtained. What before, when the computer was not used, was approximated and required a great calculus effort, now it is possible to obtain in a few minutes. Starting with this way of solving the problem it is possible to imagine other interpolation functions with other restrictions.

The shape of the camber line resulted from this mode of interpolation of the angle $\beta$ between inlet and outlet is very much alike with the one generated directly in the conformal transformation mapping plane, meaning that there are no inflexions and it is uniform increasing between inlet and outlet. This aspect suggests the possibility to couple the optimization of the blade shape through the classic method with the conformal transformation method.

The transposition of the thickness to the camber surface of the blade with the conformal transformation method gives this a very good precision compared to the classic method, and results in geometry of the blade that is much more improved.

\section{Acknowledgment}

The present work has been supported by the Romanian Government - Ministry of Education and Research, National Authority for Scientific Research through Research Grants, UEFISCDI department, project no. 21-036/2007 and project no. 21-41/2007. 


\section{References}

Anton I., Câmpian V., Carte I., (1988), Hidrodinamica turbinelor bulb şi a turbinelor pompe bulb, Editura Tehnică, Bucureşti.

Anton L.E., Miloş T., (1998), Centrifugal Pumps with Inducer, Publishing house Orizonturi universitare, Timişoara, Romania, ISBN 978-973-625-838-1.

Gülich J.F., (2008), Centrifugal Pumps, Springer-Verlag Berlin Heidelberg New York, ISBN 978-3-540-73694-3.

Gyulai Fr., (1988), Pumps, Fans, Compressors; vol I \& II, Publishing house of Politehnica University, Timişoara.

Miloş T., (2002), Computer Aided Optimization of Vanes Shape for Centrifugal Pump Impellers, Scientific Bulletin of The "Politehnica" University of Timişoara, Romania, Transactions on Mechanics, Tom 47(61), Fasc. 1, pp. 37-44, ISSN-1224-6077.

Miloş T., (2007), Method to Smooth the 3d Surface of the Blades of Francis Turbine Runner, International Review of Mechanical Engineering (IREME), Vol. 1, No. 6, pp. 603607, PRAISE WORTHY PRIZE S.r.l, Publishing House, ISSN 1970-8734.

Miloş T., (2008), CAD Procedure For Blade Design of Centrifugal Pump Impeller Using Conformal Mapping Method, Fifth Conference of the Water-Power Engineering in Romania, Published in Scientific Bulletin of University POLITEHNICA of Bucharest, Series D, Mechanical Engineering, Vol.70/2008, No. 4, ISSN 1454-2358. pp. 213-220.

Miloş T., (2009), Centrifugal and Axial Pumps and Fans, Publishing house of Politehnica University, Timisoara, ISBN 978-973-625-838-1.

Miloş T., (2009), Optimal Blade Design of Centrifugal Pump Impeller Using CAD Procedures and Conformal Mapping Method, International Review of Mechanical Engineering (IREME), Vol. 3, No. 6, pp. 733-738, PRAISE WORTHY PRIZE S.r.l, Publishing House, ISSN 1970-8734.

Miloş T., Muntean S., Stuparu A., Baya A., Susan-Resiga R., (2006), Automated Procedure for Design and 3D Numerical Analysis of the Flow Through Impellers, In Proceedings of the 2nd German - Romanian Workshop on Vortex Dynamics, Stuttgart 10-14 May 2006. pp. 1-10. (on CD-ROM).

Pfleiderer K., (1961), Die Kreiselpumpen für Flüssigkeiten und Gase, Springer Verlag, Berlin.

Radha Krishna H.C. (Editor), (1997), Hydraulic Design of Hydraulic Machinery, Avebury, Ashghate Publishing Limited, ISBN: 0-29139-851-0.

Stepanoff A. J., (1957), Centrifugal and Axial Flow Pumps, 2nd edition, John Wiley and Sons, Inc., New York.

Tuzson J., (2000), Centrifugal Pump Design, John Wiley \& Sons, ISBN 9780471361008. 


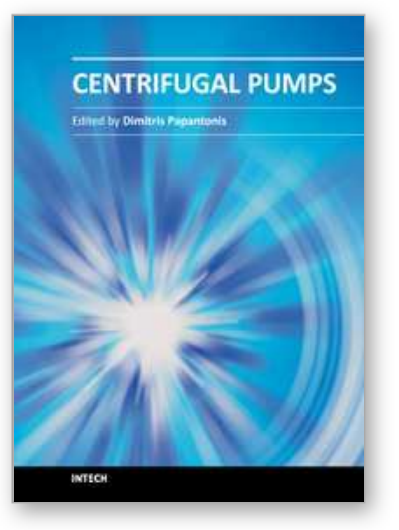

\author{
Centrifugal Pumps \\ Edited by Dr. Dimitris Papantonis
}

ISBN 978-953-51-0051-5

Hard cover, 106 pages

Publisher InTech

Published online 24, February, 2012

Published in print edition February, 2012

The structure of a hydraulic machine, as a centrifugal pump, is evolved principally to satisfy the requirements of the fluid flow. However taking into account the strong interaction between the pump and the pumping installation, the need to control the operation, the requirement to operate at best efficiency in order to save energy, the provision to improve the operation against cavitation and other more specific but very interesting and important topics, the object of a book on centrifugal pumps must cover a large field. The present book examines a number of these more specific topics, beyond the contents of a textbook, treating not only the pump's design and operation but also strategies to increase energy efficiency, the fluid flow control, the fault diagnosis.

\title{
How to reference
}

In order to correctly reference this scholarly work, feel free to copy and paste the following:

Milos Teodor (2012). Impeller Design Using CAD Techniques and Conformal Mapping Method, Centrifugal Pumps, Dr. Dimitris Papantonis (Ed.), ISBN: 978-953-51-0051-5, InTech, Available from:

http://www.intechopen.com/books/centrifugal-pumps/impeller-design-using-cad-techniques-and-conformalmapping-method

\section{INTECH}

open science | open minds

\section{InTech Europe}

University Campus STeP Ri Slavka Krautzeka 83/A 51000 Rijeka, Croatia Phone: +385 (51) 770447 Fax: +385 (51) 686166 www.intechopen.com

\author{
InTech China \\ Unit 405, Office Block, Hotel Equatorial Shanghai \\ No.65, Yan An Road (West), Shanghai, 200040, China \\ 中国上海市延安西路65号上海国际贵都大饭店办公楼405单元 \\ Phone: +86-21-62489820 \\ Fax: +86-21-62489821
}


(C) 2012 The Author(s). Licensee IntechOpen. This is an open access article distributed under the terms of the Creative Commons Attribution 3.0 License, which permits unrestricted use, distribution, and reproduction in any medium, provided the original work is properly cited. 